\title{
Characteristics of Attachment Disorder and Autism Spectrum Disorder Based on Fingertip Pulse Wave Data
}

\author{
Junko Tsujino ${ }^{1, *}$, Keiko Horii ${ }^{2}$ \\ ${ }^{1}$ Department of Children's Future, Faculty of Education, Himeji University, Himeji, Japan \\ ${ }^{2}$ Department of Early Childhood Education, Himeji-Hinomoto College, Himeji, Japan
}

Email address:

tsujino@m01.fitcall.net (J. Tsujino), cupid@rose.ne.jp (K. Horii)

${ }^{*}$ Corresponding author

To cite this article:

Junko Tsujino, Keiko Horii. Characteristics of Attachment Disorder and Autism Spectrum Disorder Based on Fingertip Pulse Wave Data. American Journal of Information Science and Technology. Vol. 4, No. 3, 2020, pp. 51-57. doi: 10.11648/j.ajist.20200403.13

Received: July 31, 2020; Accepted: August 14, 2020; Published: August 31, 2020

\begin{abstract}
DUAL measurements were taken from Child A, Child B, and Child C, respectively paired with their nursery teacher, to visualize any mental interactions with the teacher. The authors adopted finger pulse waves as a piece of biological data and used DUAL measurement to calculate mental flexibility (Largest Lyapunov Exponent: LLE) and autonomic nerve balance (ANB). Subjects are child with reactive attachment disorder (RAD; Child A), child with autism spectrum disorder (ASD; Child B), and control child (CC; Child C). As for Child A, there was correlation between the LLE of parallel measurement and the LLE of face-to-face measurement $(\mathrm{r}=0.91(\mathrm{p}=0.03))$. As for Child $\mathrm{B}$, there was correlation between the ANB of parallel measurement and the ANB of face-to-face measurement $(\mathrm{r}=0.93(\mathrm{p}=0.01))$. As for Child $\mathrm{C}$, there was a negative correlation between the ANB of parallel measurement and the LLE of parallel measurement $(\mathrm{r}=-0.89(\mathrm{p}=0.01))$. There were no changes in the mental fluctuations of RAD, despite changes in situation between parallel measurement and face-to-face measurement. It can be said that tendencies in mental fluctuations that occur by interaction with other people are constant between parallel measurement and face-to-face measurement. In conclusion, regarding RAD, this study observed mental fluctuations shown in LLE, and regarding ASD, this study observed autonomic nerve balance shown in ANB. This study observed a relationship between the autonomic nerve balance shown in ANB and the mental fluctuations shown in LLE. This led to the understanding that an interrelation of ANB and LLE is related to the communication of the control child when parallel. In terms of the mechanism of communicative ability, a mental factor is relevant for RAD, while autonomic nerve balance is relevant for ASD. In addition, it is ANB and LLE that have relevance for CC. We would like to conduct further research to track mental states of children with reactive attachment disorder and autism spectrum disorder, by using finger pulse waves data as relevant biological information.
\end{abstract}

Keywords: Attachment Disorder, Autism Spectrum Disorder, Finger Pulse Waves, DUAL Measurement, Largest Lyapunov Exponent, Autonomic Nerve Balance

\section{Introduction}

Finger pulse waves will be examined as biological information. Finger pulse waves are measured from the fingertip, making them extremely easier to obtain than electroencephalographs. In addition, a unique characteristic of pulse waves lies in the types of information that the pulse waves carry. Changes in pulse waves are closely related to physical change. Additionally, our research has been able to confirm that pulse waves are biological information that sensitively reflect not only physical but also mental states.
Also, it has become possible to "visualization" such mental states as digital information. That is, to translate psychological states into objective, quantitative data. In addition, finger pulse waves carry heart rate information. Activities of the sympathetic nervous system and parasympathetic nervous system can be measured by analyzing this heart rate information, using a special method called spectrum analysis. The level of activity of the sympathetic nervous system rises when the mind and body are active, while the level of activity of the parasympathetic nervous system rises when the mind and body are resting to recover from exhaustion. In addition, the quantitative information of the activities of these two 
nervous systems can be used to calculate autonomic nerve balance, which is an index showing which of these nervous systems has predominance in terms of level of activity. [1, 2]

Finger pulse waves can be examined as biological information to measure the characteristics of an individual and over-time changes thereof.

The authors have repeatedly studied fingertip pulse waves. [3-9].

There was a change in the parallel and face-to-face measurements of LLE in children with attachment disorder, but no change in children with autism spectrum. ANB shows that children with autism have higher face-to-face measurements than parallel measurements. [9] And, in a study of autism spectrum disorders, LLE was found to be significantly higher in children with autism spectrum disorders than in controls. [6]

This study deepens the research on attachment disorder and autism spectrum disorder based on the verification by these past fingertip pulse waves.

When children are subjected to their parents' abuse or neglect, these experiences hinder the formation of attachment resulting later in the child's inability to form sufficient interpersonal relationships $[10,11]$. This leads to the development of attachment disorder. People who suffer from attachment disorder have difficulty gaining objects of attachment, due to past experiences that threatened their basic safety. Attachment disorder could be considered a disorder caused by the absence of formation of attachment between the child and his or her primary caregivers. Attachment disorder is considered the cause of many and various, long-term disadvantages in a person's ability to build relationships and gain happiness through life in society.

In recent years, there has been an increasing interest in "preschoolers with special educational needs in early childhood education" at nursery schools and kindergartens. In relation to this fact, a number of articles regarding "anxious" children is increasing. This is because they do not know how to optimally treat "anxious" children in group activities of childcare centers and kindergartens and therefore researches and empirical studies are demanded.

S. Shimano (2007) affirmed that in general, many "anxious" children exhibit common behavioral characteristics with children with mild developmental disorder [12]. While no definition has been established for the term "mild developmental disorder," it may be commonly considered as similar to developmental disorder in the society [13].

In childcare centers and kindergartens, children with autism spectrum disorder draw special attention among those with developmental disorders. The three signs peculiar to autism spectrum disorder are: (1) "social impairment," (2) "language and communication disorder," and (3) "restricted interest and obsession to a specific event."

In addition, attachment disorder also requires further researches and actions. Attachment disorder is a generic term for disorders caused by inability to construct a stable attachment relationship with rears due to long-term abuse during babyhood or childhood, parent's death, or another cause. A relationship with loved ones can considerably affect child's independence.
Child's confidence in constant protection from the loved ones can foster his/her independence. Attachment disorder can manifest symptoms very similar to those of autism disorder spectrum. Nonetheless, developmental disorder is considered usually not affected by the child's growing environment, while attachment disorder is acquired after birth. But reportedly, attachment disorder can be misrecognized as autistic spectrum because they are indifferent to others.

Based on these understandings, children's behaviors were evaluated using "Checklist for Anxious Children" in advance, and then DUAL measurements of finger pulse waves were taken from children and their nursery teacher.

The purpose of this study is to visualize mental interactions between disorder children and their nursery teacher by biological information.

While attachment disorder is an acquired disorder caused by factors related to the human environment in which the child is raised, autistic spectrum disorder is a congenital disorder of the brain. Despite these causal differences, one being congenital and the other developmental, patients of these two disorders share one common trait. Namely, they experience difficulty forming interpersonal relationships. This study will examine how this condition is manifested as biological information in terms of mental fluctuation and autonomic nerve balance. Characteristics of the control child will also be examined in relation to this.

\section{Attachment Theory and Diagnosis of Autism Spectrum Disorders}

\subsection{Attachment Theory}

Attachment issues in mother-child relationships have been theorized since the 1960s. Bowlby, J. (1969) first developed an attachment theory to explain why children experience physical/mental pain when they are separated from their mothers [14]. After focusing on infancy and early childhood, Bowlby began to shift focus to these children's adulthood. Attachment formation during early childhood is an experience that significantly influences the rest of that person's life. In particular, attachment regains a significant role in the dynamic process of the second separation-individuation during early adulthood. In general, a person first develops concepts of the self and others in conjunction with early experiences of attachment. These concepts, which are related to internal representation, create mental comfort in the individual's daily life. They also influence the further deepening and expansion of attachment, along with mind-and-body development.

Ainsworth, M. S., who had participated in Bowlby's research for a time, later classified attachment behavior into three types using an experiment called the "Strange Situation" procedure [15].

Later on, Main, M. and Morgan, H. (1996) reported their discovery of a "disorganized/disoriented attachment behavior ('D' classification)" that did not fit into the classical three classifications of attachment observed through the Strange 
Situation procedure by Ainsworth and others [16]. Main and Morgan pointed out a similarity between the state of the children of this fourth classification and the state of dissociation. The parents of children exhibiting this type of behavior are characterized by frightened behavior or behavior that frightens their children. The behavior of the parents and the behavior of the children are related to the internal working model of attachment. The internal working model has an important function as an unconscious, individual-specific, psychological framework for the organization of diverse information related to attachment, and for the progress of systemization of attention, memory, emotions, and actions.

In addition, the internal working model is constructed based on past interactions, and it is an abstract and generalized psychological representation related not only to specific transactions with the caregiver, but also to one's surrounding world and self [17]. Studies have hypothesized that we use this internal working to interpret the world around us, predict the behavior of others, and plan our own actions.

\subsection{Diagnosis of Autism Spectrum Disorders}

The history of autism spectrum began in 1943 with the report on the classical, so-called Kanner-type of autism [18]. The causes of this condition, around this period, were considered care-related and environmental. Later in 1968, Rutter announced a linguistic-cognitive disorder theory, and this inspired many others to perform cognitive psychological research on ASD [19]. In 1980, a diagnostic category called pervasive developmental disorders was included in the third edition of Diagnostic and Statistical Manual of Mental Disorders (DSM), which is a publication that lists the standard, international criteria for diagnosing mental disorders [20]. In 1981, Wing and others reported on Asperger syndrome, and Asperger disorder was included in the fourth edition of DSM in 1994 [21, 22]. As a result, types of ASD that did not involve retardation of linguistic development or intellectual functions were included in diagnoses, while these had not been widely recognized until then. Progress in brain science and technology in the recent years has resulted in the accumulation of much discoveries and understandings about brain abnormalities of ASD and related cognitive psychological hypotheses. This has led to a general consensus that ASD is caused not by carerelated, environmental factors but by organic abnormality of the brain. Further, the diagnostic criteria underwent revision with the publication of the fifth edition of DSM in 2013, and the name of the disorder changed from pervasive developmental disorders to autism spectrum disorder. This was accompanied by the removal of the formerly classified items such as autistic disorder, Asperger disorder, and unspecifiable pervasive developmental disorder. Social communication disorder limited, repetitive behavior, and other conditions became the necessary criteria for ASD diagnosis. In addition, the clinical applicability of the diagnostic criteria was improved by requiring three-tier assessments of severity based on need for support for social communication disorder and limited, repetitive behavior respectively; by recognizing attention-deficit hyperactivity disorder as comorbidity; and by the inclusion of notes prompting consideration for latent ASD. [23]

\section{Largest Lyapunov Exponents and Autonomic Nerve Balance}

The chaotic analysis of the data obtained from the pulse wave of the fingertip was performed to calculate Largest Lyapunov Exponent (LLE) and autonomic nerve balance (ANB) [24, 25].

\section{Methods}

\subsection{Measurement Method for Fingertip Pulse Wave}

A finger cuff with a built-in, infrared sensor is attached to the fingertip, to measure changes in levels of hemoglobin running through capillary vessels. This information is then digitized and stored in a computer. A common type of data transfer cable, such as a USB cable, is used to connect the finger cuff to the computer. In addition, the computer is an ordinary, consumer laptop with no special specifications. However, on the computer is software specialized for analysis. Using the above equipment, finger pulse waves are saved as digital data, nonlinear analysis is conducted on them, and the results are outputted. (see Finger 1.)

\subsubsection{Period of Measurement for Fingertip Pulse Wave}

Fingertip pulse wave measurements were taken in April, May, and June 2019.

\subsubsection{Place of Measurement}

A certified child center in Hyogo Prefecture, Japan

\subsubsection{Tools Used in the Research/Study}

Measurement Device for Finger pulse waves

Lyspect 3.5 [Chaos Technology Research Laboratory] was used.

\subsubsection{Diagnostic Criteria and Number of Measurements}

The RAD child and The ASD child diagnosed with DSM-V diagnostic criteria by two Japanese Society of Child and Adolescent Psychiatrists. The RAD child measured 5 times and the ASD child measured 6 times. One child of the same age, who had no physical illness and had no apparent developmental problems, was used as a control child. The age of three children is five.

\subsubsection{How Finger Pulse Waves Was Measured}

The room temperature was moderate. DUAL measurements were taken for three minutes from a child and his/her nursery teacher, who sat on a chair with open eyes, wearing a cuff on the tip of the second finger of the left hand.

First time: Parallel measurement (the child and the nursery teacher sit side by side)

Second time: Face-to-face measurement (the child and the nursery teacher face each other and shake hands with the right hand, and the nursery teacher speaks quietly to the child.) 


\subsection{The Check Lists for Anxious Children}

"Checklist for anxious Children" was developed by Shimano (2014). "Troubles (anti-social behaviors)," "dissocial behaviors," "autism behaviors," and "hyperactive behaviors" can be evaluated and scored. Percentages to the maximal score were calculated as evident rates for four subscales. Shimano stated, "If the evident rate is $50 \%$ higher, the behavior can be characteristic to that of anxious child."

"Checklist for anxious Children" was filled by their (female) nursery teacher on the day before measurement of finger pulse waves. It was filled for 42 children ( 21 boys and 21 girls).

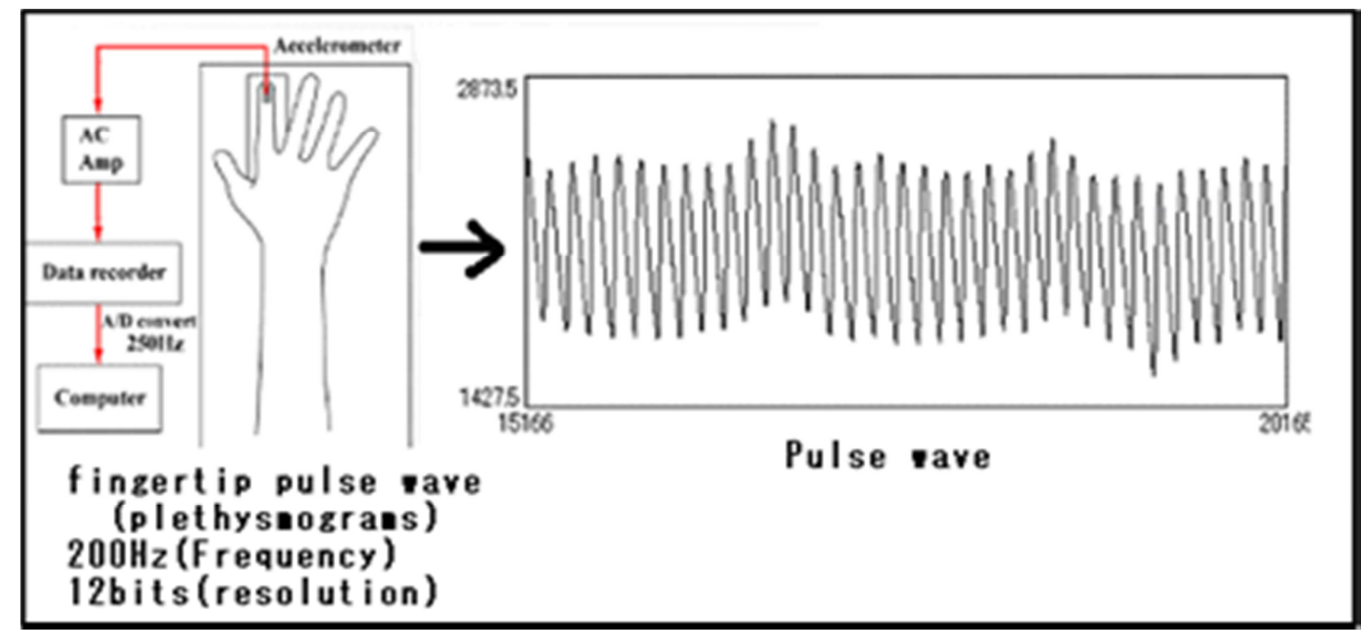

Figure 1. Measurement method of finger pulse wave.

\section{Analysis and Result}

Statistical analysis software JMP (SAS Institute Inc.) was used for data analysis.

Data was analyzed as follows: Table 1 shows mean (SD), median and range of LLE and ANB. Table 2 shows the correlation coefficients for LLE and ANB.

As for Child A, there was correlation between the LLE of parallel measurement and the LLE of face-to-face measurement $(\mathrm{r}=0.91(\mathrm{p}=0.03)$, see Figure 2$)$. As for Child B, there was correlation between the ANB of parallel measurement and the ANB of face-to-face measurement $(r=0.93(p=0.01)$, see Figure 3). As for Child $C$, there was a negative correlation between the ANB of parallel measurement and the LLE of parallel measurement $(\mathrm{r}=-0.89$ $(p=0.01)$, see Figure 3$)$. There were no changes in the mental fluctuations of RAD, despite changes in situation between parallel measurement and face-to-face measurement. It can be said that tendencies in mental fluctuations that occur by interaction with other people are constant between parallel measurement and face-to-face for measurement.

There were no changes in the autonomic nerve balance of ASD, despite changes in situation between parallel measurement and face-to-face measurement. This led to the understanding that the tendencies in autonomic nerve balance, which is affected by stress, are constant between parallel measurement and face-to-face measurement.

In addition, the ANB of parallel measurement and the LLE of parallel measurement had relevance regarding $\mathrm{CC}$. The higher the ANB, the lower became the LLE. Mental fluctuations were small when the level of activity of the sympathetic nervous system was high, while mental fluctuations were moderate when the level of activity of the sympathetic nervous system was low.

Consideration of the characteristics of RAD, ASD, and CC from the viewpoint of LLE and ANB led to the understanding that it is LLE that has relevance for RAD, while it is ANB that has relevance for ASD. In addition, it is ANB and LLE that have relevance for $\mathrm{CC}$.

It can be said that there is no difference in the evaluation scores of the child of RAD and ASD on the four checklist subscales. In behavioral assessment, the child with RAD and the child ASD have similar behavioral characteristics. (see Figure 5 and Figure 6)

\section{Conclusion and Remark}

In conclusion, regarding $\mathrm{RAD}$, this study observed mental fluctuations shown in LLE, and regarding ASD, this study observed autonomic nerve balance shown in ANB. In addition, regarding $\mathrm{CC}$, this study observed a relationship between the autonomic nerve balance shown in ANB and the mental fluctuations shown in LLE. This led to the understanding that an interrelation of ANB and LLE is related to the communication of the control child when parallel. In terms of the mechanism of communicative ability, a mental fluctuation factor is relevant for RAD, while autonomic nerve balance is relevant for ASD.

Further consideration is necessary to determine if these relevant factors were observed because RAD is an acquired disorder and ASD a congenital disorder.

Through visualization of mental interactions between children and others, it becomes possible to know children's mental state. In particular, knowing mental state of children with RAD or ASD can give us a clue to develop a human relationship with them.

In an effort to scientifically understand the relationship 
between behavior in children and their biological signals, we measured the finger pulse waves of children and carried out chaos analysis of the measured data. We think that our analysis will contribute to the prevention of behavioral problems in children and encourage the adoption of early countermeasures.

We would like to conduct further research to track mental states of children with RAD, ASD and anxious children, by using finger pulse waves data as relevant biological information.

Further research will require accumulation of data from many children with $\mathrm{RAD}$ and $\mathrm{ASD}$, in order to improve validity and reliability. Development of a universal theory will also be aimed for. In addition, a longitudinal study over a long period of time is planned for, given the possibility that the physical and psychological states of children with the disorders can change through stages of growth.

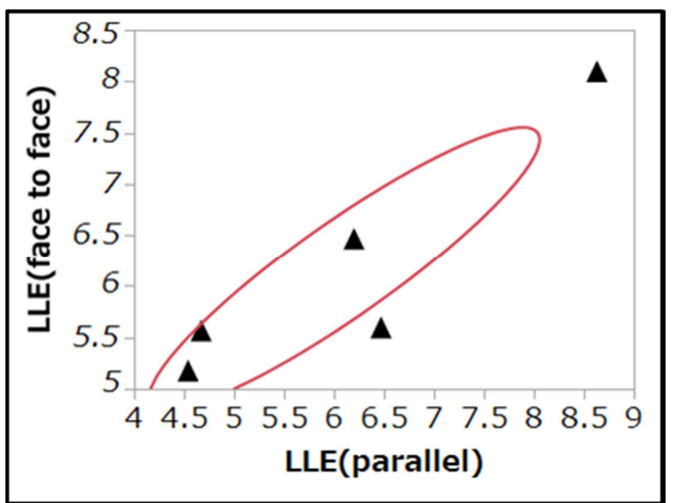

Figure 2. Relationship between LLE (parallel and face to face) on RAD.

Table 1. Mean value (SD), Median, Range of LLE and ANB.

\begin{tabular}{llll}
\hline & RAD (N=5) & ASD (N=6) & CC (N=7) \\
\hline LLE (parallel) & & & $4.98(1.00)$ \\
Mean value (SD) & $6.10(1.66)$ & $5.20(1.10)$ & 4.91 \\
Median & 6.20 & 5.46 & $3.56-6.58$ \\
Range & $6.54-8.63$ & $3.29-6.31$ & $5.29(1.06)$ \\
LLE(face to face) & & & 5.11 \\
Mean value (SD) & $6.17(1.17)$ & $5.06(1.08)$ & $3.95-7.35$ \\
Median & 5.59 & 5.11 & $5.90(2.37)$ \\
Range & $5.17-8.09$ & $3.55-6.48$ & 5.57 \\
ANB (parallel) & & & $2.05-9.04$ \\
Mean value (SD) & $6.29(1.12)$ & $4.418(1.25)$ & $5.93(1.68)$ \\
Median & 6.93 & 4.42 & 6.64 \\
Range & $4.50-7.11$ & $2.26-5.71$ & $3.84-7.96$ \\
ANB (face to face) & & $4.81(1.62)$ & \\
Mean value (SD) & $5.89(1.39)$ & 4.96 & $2.23-7.11$ \\
Median & 6.37 & & \\
Range & $4.40-7.55$ & & \\
\hline
\end{tabular}

Table 2. Correlation coefficient for LLE and ANB.

\begin{tabular}{llll}
\hline & Correlation coefficient & & \\
\cline { 2 - 4 } & RAD & ASD & CC \\
\hline LLE (parallel and face to face) & $0.91(\mathrm{p}=0.03)$ & $0.75(\mathrm{p}=0.08)$ & $-0.36(\mathrm{p}=0.43)$ \\
ANB (parallel and face to face) & $0.86(\mathrm{p}=0.06)$ & $0.93(\mathrm{p}=0.01)$ & $0.65(\mathrm{p}=0.12)$ \\
ANB (parallel) and LLE (parallel) & $0.26(\mathrm{p}=0.26)$ & $0.67(\mathrm{p}=0.14)$ & $-0.89(\mathrm{p}=0.01)$ \\
ANB (face to face) and LLE (face to face) & $0.55(\mathrm{p}=0.34)$ & $0.78(\mathrm{p}=0.07)$ & $0.29(\mathrm{p}=0.52)$ \\
\hline
\end{tabular}

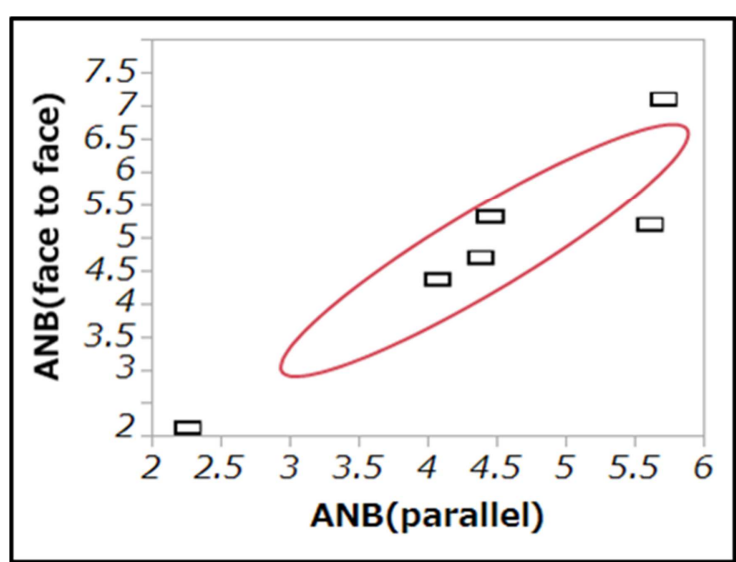

Figure 3. Relationship between ANB (parallel and face to face) on ASD.

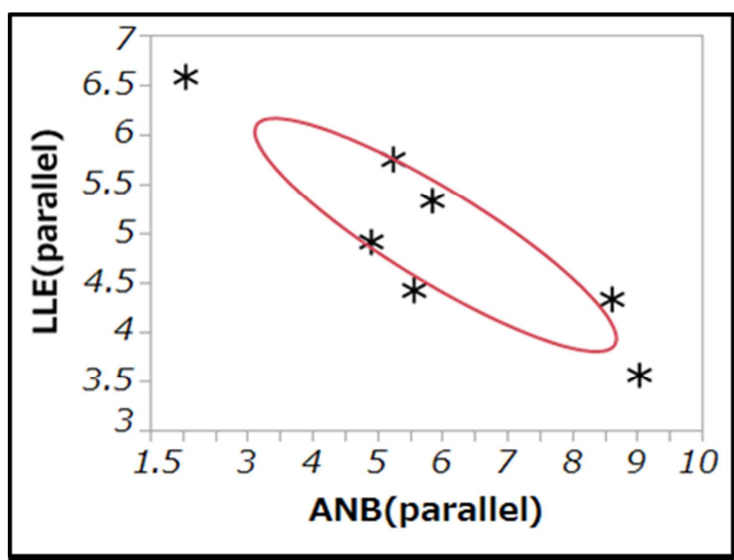

Figure 4. Relationship between ANB (parallel) and LLE (parallel) on CC. 


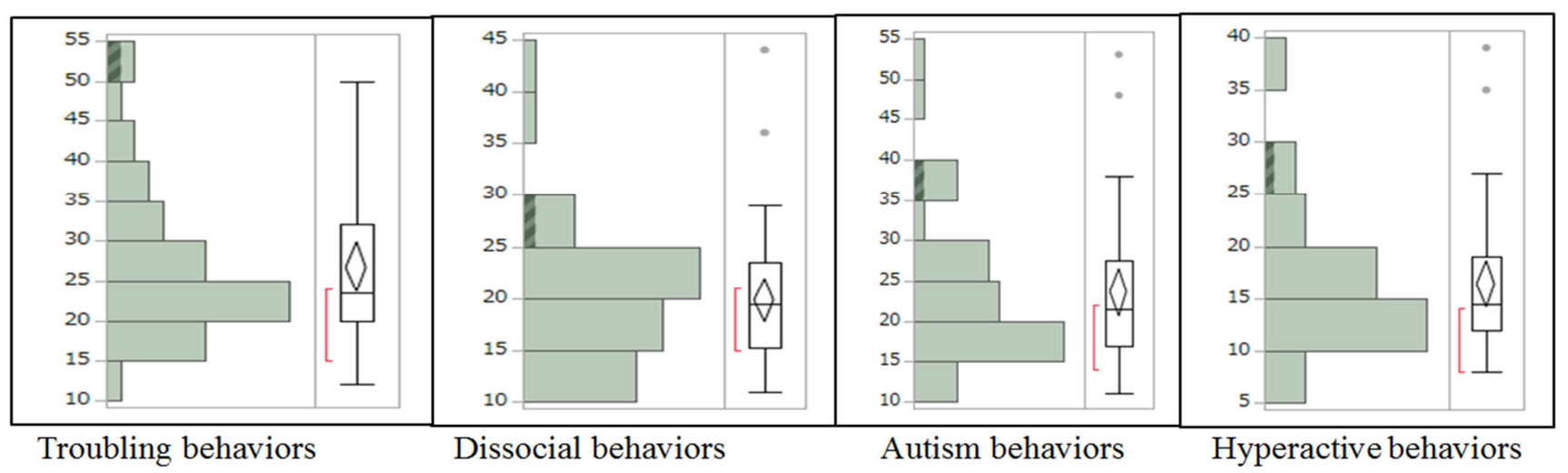

Figure 5. Percentage of the number of children four subscales on behavioral characteristics of children (mark: child A).

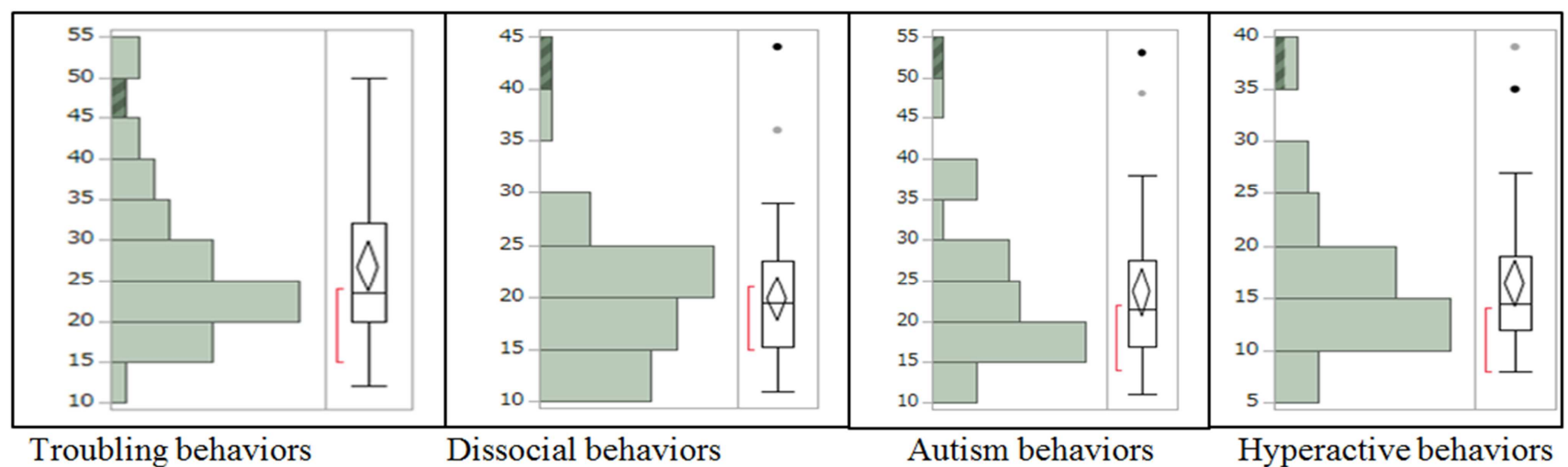

Figure 6. Percentage of the number of children in four subscales on behavioral characteristics of children (mark: child B).

\section{Acknowledgements}

We are truly grateful to the parents of the children in the Himeji Himawari preschool for their cooperation in the finger pulse waves measurements.

\section{References}

[1] Oyama-Higa, M. (2006) Development of a self-check system for mental health, which uses Nonlinear Chaos Analysis of Pulse Waves. The 12th Open Symposium "Humanities Science and Database" Executive Committee, 31-41.

[2] Oyama-Higa, M. (2012) Psychology of "fluctuation" that enhances the immunity of the mind. Shoudensha, Japan.

[3] Tsujino, J. and Oyama-Higa, M. (2007) Does a Mother's Attachment to Her Child Affect Biological Information provided by the Child? 2007 IEEE Conference on Systems, Man, and Cybernetics 2030-2034.

[4] Tsujino, J. and Oyama-Higa, M. (2008) Measurement of Ear Pulse Waves in Children: Effect of Facing Another Child and Relationship to an Action Index. 2008 IEEE International Conference on Systems, Man and Cybernetics Proceedings, 2972-2976.

[5] Tsujino, J. and Oyama-Higa, M. (2010) Examining the relationship between the child's pulse waves and mother's attachment. Journal of Kansai Women's College, 19: 13-26.
[6] Tsujino, J. and Ue, H. (2011) Examining the relationship between ego developmental crisis and fingertip pulse waves in university students. Journal of Kansai Welfare Sciences, 2: 115-124.

[7] Tsujino, J. and Tanabiki, M. (2017) Characteristics of children's "anxiety" behavior in chaos analysis of fingertip pulse waves. Himeji-Hinomoto College, 40: 97-106.

[8] Tsujino, J., Oyama-Higa, M. (2018) Longitudinal Study on Mental Interactions between Difficult Children and their nursery teacher based on DUAL measurements of Finger Pulse Waves. ICIME2018 International Conference, 94-104.

[9] Tsujino, J. and Horii, K. (2019) Child attachment disorder and biological information. Bulletin of Himeji-Hinomoto College 42: 99-109.

[10] Zeanah CH, Gleason MM (2015) Annual research review: Attachment disorder in early childhood -clinical presentation, causes, correlates, and treatment. Journal of Child Psychology Psychiatry, 56: 207-222.

[11] Lehmann. S, Havik, OE. \& Havik, T. et al. (2013) Mental in foster children: A study of prevalence, comorbidity and risk factors. Child Adolescent Psychiatry Mental Health, 7: 39.

[12] Shimano, S. (2014) Study (7) on "anxious children": check lists are made based on surveys of "difficult children" in kindergartens. Bulletin of Morioka Junior College, 24: 33-44.

[13] Shimano, S. (2007) A study of difficult children: research on the difficult child as observed in preschools by students of a junior college. Bulletin of Morioka Junior College 17: 21-35. 
[14] Bowlby, J. (1969) Attachment and Loss Vol.; Attachment. Basic Books.

[15] Ainsworth, MD., Bell, SM (1970) Attachment exploration and separation: Illustrate $4^{\text {th }}$ by the behavior of one-year-olds in a strange situation. Child Development, 4: 49-67.

[16] Main, M. \& Morgan, H. (1996) Disorganization and disorientation in infant strange situation behavior - Phenotype resemblance to dissociative states, In (eds.) Michelson, Lk. \& Ray W. J.: Theoretical empirical and clinical perspective. Plenum Press, New York, 107-138.

[17] Bretherton, I. (1990) Communication patters internal working models, and intergenerational transmission of attachment relationships. Infant Mental Health Journal, 11: 237-252.

[18] Kanner, L. (1943) Autistic disturbances of affective contact. Nervous Child, 2: 217-250.

[19] Rutter, M. (1968) Concepts of autism: a review of research. Journal of Child Psychology \& Psychiatry, 9: 1-25.
[20] Diagnostic Statistical Manual of Mental Disorders THIRD EDUTION; Dsm-3 (1980) American Psychiatric Association.

[21] Wing, L. (1981) Asperger's syndrome: a clinical account. Psychological Medicine, 11 (1): 115-29.

[22] Diagnostic Statistical Manual of Mental Disorders FORTH EDUTION; Dsm-4 (1994) American Psychiatric Association.

[23] Diagnostic Statistical Manual of Mental Disorders FIFTH EDUTION; Dsm-5 (2013) American Psychiatric Association.

[24] Shimizu, T. and Miao, T. (2004) Evaluations of Driver's status by chaotic analysis of finger photoplethysmography. human interface 6 (1): 97-99.

[25] Sano, M., Sawada, Y. and Iwanaga, I. (1992) Chaotic pulsation in capillary vessels and its dependence on mental and physical conditions, Int. J. Bifurcation and Chaos, 2: 313-324. 